\title{
Bioanalytical strategies for in-vitro and in-vivo evaluation of the toxicity induced by metallic nanoparticles
}

Jose L. Luque-Garcia ${ }^{1}{ }^{*}$, Raquel Sanchez-Díaz ${ }^{1}$, Isabel Lopez-Heras ${ }^{1}$, Pilar Martin ${ }^{2}$ and Carmen Camara $^{1, *}$

${ }^{1}$ Department of Analytical Chemistry. Faculty of Chemistry. Complutense University of Madrid. Av. Complutense s/n, 28004, Madrid, Spain

${ }^{2}$ Department of Vascular Biology and Inflammation, Fundación Centro Nacional de Investigaciones Cardiovasculares Carlos III, Madrid, Spain E-28029.

Tel: +34913944318; Fax: +34913944329

* Corresponding author: ccamara@quim.ucm.es and jlluque@quim.ucm.es

\begin{abstract}
The increasing use of metallic nanoparticles (MNPs) in a wide variety of applications have consequently lead to an urgent need to evaluate the impact of these new materials on human health and the environment. To date, the potential toxicity of MNPs as well as their interaction mechanisms with cells and living organisms have not been fully addressed. In this article, we discuss the different bioanalytical strategies that have been used so far with this purpose. We consider different methods aim to evaluate cellular uptake and localization in cells and tissues, and in vitro methods for the study of the toxicity induced by MNPs considering different toxicity markers and high throughput approaches for the identification of specific targets involved in the cell-MNPs interaction. Special strategies related to the use of animal models to assess in-vivo toxicity of MNPs are also discussed.
\end{abstract}

Keywords: Metallic nanoparticles. Toxicity assessment. In-vitro and in-vivo assays 


\section{Introduction}

In the last years, nanoparticles (NPs) have received a great attention for their use and applicability in many new consumer products. A recent estimate suggests that more than 1000 NP-containing consumer products are currently on the market. In addition, their ability to advance science with novel analytical and medical tools also make them relevant to both physical and life sciences [1].

NPs are generally defined as spheres with a diameter between 1 and $100 \mathrm{~nm}$. This small size gives them unique properties, especially because their specific surface area is larger and their reactivity is increased or different compared to bulk materials. These specific features are often linked with a potential toxicity [2]. Among the different kinds of NPs, metallic nanoparticles (MNPs) are being particularly used in many applications. The products and applications of MNPs include electronics, optics, textiles, medical applications, cosmetics, food packaging, water treatment technology, fuel cells, catalysts, biosensors and agents for environmental remediation [3, 4]. As a result of these applications, exposure of MNPs to the environment and humans are becoming increasingly widespread. Consequently, different metals in the form of MNPs have gained an increasing access to tissues, cells and biological molecules within the human body [5]. Human exposure to MNPs occurs most likely during manufacturing processes, but inhalation of MNPs released to the atmosphere, ingestion of water or food containing MNPs and dermal exposure from various sources such as body lotions and sunscreen is possible [6].

To date, the impact of MNPs exposure to human health and the environment is not fully assessed [7]. Research efforts to assess the toxic potential of MNPs have presented some serious and far-reaching challenges and there remains an urgent need for well-designed studies that will generate data so that risk assessments for MNPs can be conducted [8].

The chemical composition, shape, size, stability, surface coating, functionalization as well as the purity of the MNPs become essential for application purposes but also for their potential toxicity [9]. Particle chemistry is especially relevant; depending on their chemistry, NPs can show different cellular uptake and ability to catalyze the production of reactive oxygen species, which is directly related with toxicity [10]. Many studies have also suggested the importance of shape and crystallographic phase when evaluating the toxicity induced by MNPs $[3,11]$. The uptake and cellular distribution of NPs are greatly influenced by their size. It has been demonstrated that cellular internalization is more pronounced for small-sized NPs as compared to equivalent high-sized NPs at the same dose [12]. In addition, small MNPs show higher reactivity because of their higher surface area [2]. Aggregation of MNPs mainly depends on surface charge, material type and size. The formation of MNPs aggregates affects the degree of cell internalization and thus modifies the potential toxic effect [13]. It is also crucial to consider surface modification of NPs, either by proteins or ions present in the culture media or when using metals, organic molecules or polymers as surface coatings $[12,13,14]$. These modifications can drastically change the physicochemical properties of MNPs, including their magnetic, electric, and optical properties as well as their chemical reactivity [15]. 
In order to correlate any toxic reaction with a MNP type, it is indispensable to investigate if the nanoparticles are attached to the cell surface or if they enter cells. If NPs are found in cells, their localization in different compartments such as endosomes, lysosomes, mitochondria, the nucleus or the citosol, may also provide some answers regarding their potential toxicity [16]. It is also of great importance the understanding of the complex processes that govern cellular uptake and intracellular fate of MNPs [17]. Finally, in vivo studies are also needed to design specific and safe clinical trials to develop new strategies for the applied use of MNPs and also to evaluate the risk to the environment [18].

In this review, we present different analytical and bioanalytical strategies to study the interaction of MNPs with cells and living organisms in order to assess their potential toxicity. We start by discussing different alternatives to evaluate cellular uptake and localization of MNPs by using atomic techniques and imaging approaches. In vitro assays to study the interaction of cells with MNPs are also reviewed considering both; those that aim to evaluate specific cellular pathways or cellular responses to MNPs exposure and those based on high throughput discovery platform focused on the identification of particular targets (genes and/or proteins) involved in the cell-MNPs interaction. Finally, considerations about in vivo assays depending on the different animal models that have been used to date are also reviewed.

\section{Cellular uptake and localization of MNPs}

When studying the interaction of MNPs with living organisms and cells, one of the key aspects is the evaluation of the cellular uptake and the localization of the MNPs within the cells and tissues. The quantification of MNPs and ions released from NPs is mainly carry out by sensitive analytical techniques such as inductively coupled plasma-mass spectrometry (ICP-MS), while the localization, which requires spatial resolution analysis, is generally performed by different microscopic techniques.

\subsection{Quantification of NPs and ions released from NPs using spectrometric techniques}

ICP sources are regarded as excellent tools for elemental analysis. Many authors have used techniques based on ICP in order to provide a quantitative analysis of the uptake process of MNPs in different organisms [19]. ICP combined with mass spectrometric detection (ICP-MS) provides high sensitivity and robustness for quantifying the metal composition from a large variety of samples. Christen and Fent used an ICP-MS equipped with an octopole reaction system to minimize possible interferences, and confirmed that the cytotoxicity induced by Ag-doped $\mathrm{SiO}_{2}$ NPs was caused by Ag ions released from the NPs [7]. Other detectors have also been coupled to an ICP source for this purpose. The use of ICP with optical emission spectroscopy detection (ICP-OES) has been employed to perform cadmium analysis as an indication of the concentration of CdSe/ZnS quantum dots (QDs) NPs in plasma and organs of Daphnia magna after $48 \mathrm{~h}$ of exposure [20]. The impact of three metal oxide NPs (nonporous $\mathrm{SiO}_{2}$, mesoporous $\mathrm{SiO}_{2}$ and anatase $\mathrm{TiO}_{2} \mathrm{NPs}$ ) on the immune response of mast cells was evaluated using ICP-OES, showing that the interaction cell-NPs depended on the chemically modified surface of the NPs [21].

However, the direct analysis of MNPs using ICP as ionization source present some drawbacks due to the limited availability of MNPs commercial standards and the low nebulization efficiency. This 
technique is able to ionize and atomize spherical NPs in the range of 5-25 nm, in aqueous and organic solutions [22]. However, the presence of MNPs aggregates might not be effectively ionized in the plasma, which can result in lower recoveries. In these cases of low ionization efficiency, electro-thermal vaporization (ETV) should be considered as an alternative sample introduction system because it allows for pre-digesting the MNPs [23, 24]. Another problem that has to be considered is the potential adsorption of the MNPs on the sample introduction system, which can complicate the quantification of MNPs in real samples [25]. Thus, it is important to carry out a previous digestion, using the combination of both acids and high temperature [26], to determine the total metal concentration of the tested element. In this way, the concentration of $\mathrm{Cd}$ and Se were determined in Arabidopsis thaliana plants exposed to $\mathrm{CdSe} / \mathrm{ZnS}$ QDs. Leaves and roots were thoroughly rinsed with Milli-Q water to remove material that was neither adsorbed nor integrated by the plant. The tissues were digested in concentrated nitric acid and $30 \%$ hydrogen peroxide (4:1) for 2 hours using a hot plate [27]. Conventional digestion procedures can be very time-consuming so the use of microwaves can minimize the time required for sample treatment. In addition, a closed digestion microwave vessel might lower the risk of analyte losses in comparison to open digestion procedures. The accumulation of $\mathrm{TiO}_{2} \mathrm{NPs}$ was observed in lung tissue of Wistar rats after analysis of the levels of $\mathrm{Ti}$ by digesting the sample with nitric acid and hydrogen peroxide prior to double focusing (DF)-ICP-MS detection [28]. Differences between the bioaccumulation of Ag NPs and Ag microparticles in rat brain microvessel vascular endothelial cells (BMVECs) were evaluated by acid digestion with nitric acid prior to the analysis with ICP-MS. This study demonstrated that Ag NPs are accumulated into cells more easily that microparticles [29].

Analytical methods based on acid digestions offer the possibility to reduce the matrix effects due to the elimination of biological components present in the sample or stabilizing agents employed to avoid NPs aggregation. However, this procedure makes impossible to distinguish between metal species (MNPs uptaked by the organism and other metal species). An alternative to digestion of solid samples is the use of ultrasounds. The use of ultrasound-assisted extraction has been shown as a very promising methodology for the extraction of both metal species and MNPs. In addition, the use of ultrasonic energy breaks existing MNPs aggregates [30]. However, as in the digestion procedures, alteration of the chemical form of MNPs might occur due to the use of basic or acid solutions in the extractant. Using this approach, the total $\mathrm{Ti}$ concentration was determined in fibroblasts exposed to $\mathrm{TiO}_{2} \mathrm{NPs}$ after sonication of trypsinized cells in an ultrasonic bath for $30 \mathrm{~min}$ [11].

Other techniques including furnace atomic absorption spectrometry (FAAS), which has been used to determine the total Ag content in harvested THP-1 cells prior acid digestion in $69 \% \mathrm{HNO}_{3}$ [31], or graphite furnace atomic absorption spectrometry (GFAAS) [13] have been used as alternative to ICPbased techniques.

Despite the numerous advantages offered by ICP-MS and other atomic techniques, it is important to emphasize that this technique by itself is unable to differentiate between metal species (MNPs, ions, etc.). Previous treatments such as filtration, centrifugation and sedimentation that allow isolation of MNPs from solutions, or otherwise, the use of a separation technique coupled to the ICP-MS, are required. The use of separation techniques such as liquid chromatography or field flow fractionation coupled to ICP-MS are among the most promising approaches. In this term, size exclusion 
chromatography (SEC) columns and hydrodynamic chromatography (HDC) have been described in the literature as alternatives to separate MNPs of sizes ranging from 10 to $80 \mathrm{~nm}[32,33]$. Currently, field flow fractionation (FFF) is a promising method for size separation of natural and engineered NPs, providing a wide dynamic range. Although an ultraviolet (UV) detector is the most common system used in combination with a FFF, elemental information can be obtained combining FFF with ICP-MS. One of the main advantages of coupling FFF-ICP-MS is not only the possibility for multiple elemental detection, but also the higher sensitivity achieved (in the range of $n \mathrm{~L}^{-1}$ ) as compared to $\mathrm{UV}$ detection. To provide complementary information, the FFF can also be coupled to a multiangle light scattering (MAL) for particle size characterization. The two mainly used FFF principles are the sedimentation (SedFFF) and the asymmetric flow field flow fractionation (AF4). SedFFF-ICP-OES has been employed to study sizebased elemental distribution of air particles and MNPs, such as Al, Ti and Fe [34].

In recent investigations about both the toxicity of $\mathrm{TiO}_{2}$ present in sunscreen products and the influence of polymer-coated CdSe/ZnS QDs to Daphnia magna, the on-line coupling of FFF to ICP-MS was applied to characterize the particle size as well as the composition of MNPs [20, 35]. In spite of the promising results obtained so far, there are still some aspects that require further investigation such as problems related to membrane-particle interaction, different behavior of MNPs according to their chemical structure, and the influence of eluents during the separation step, which can lead to erroneous size information and negatively affect on recovery rates.

\subsection{Determining the stability of MNPs}

The aggregation state of MNPs is a crucial factor for experimentally assessing their toxic activity. In in vitro studies, the influence of biological components present in the culture media on MNPs has been evaluated in many occasions. It is known that serum proteins, antibiotics, vitamins, electrolytes, etc., may interact with MNPs changing their physicochemical properties and aggregation state. While serum proteins may absorb onto the MNPs surface changing their size and surface chemistry, the presence of electrolytes and the high ionic strength of the culture media may alter their aggregation state. Due to the dimensions of the MNPs, the detection and characterization of MNPs is generally performed by microscopic techniques. Scanning electron microscopy (SEM), transmission electron microscopy (TEM) and atomic force microscopy (AFM) are the most commonly used techniques to visualize MNPs and their features including the aggregation state, dispersion, sorption and particle size. Before evaluating the toxicity of Ag NPs in different cell lines, Mukherjee et al. determined the Ag NPs stability and its possible interaction with the culture media by TEM and SEM [5]. The aggregation state of Ag NPs was also studied by Oukarroum et al. where TEM images showed an increase in the diameter of the NPs in all the culture media tested for growing two types of green algae [37]. Although TEM images provide useful information about the morphology and aggregation state of MNPs, these images might not completely represent the morphology of the MNPs under the real experimental conditions because TEM images are acquired on dried samples. As an alternative, dynamic light scattering (DLS) can be used combined with TEM, thus allowing the analysis of the MNPs to be performed in solution. The characterization of stable monodisperse and aggregated Si NPs by TEM and DLS was carried out to study the effect of these MNPs on macrophages and fibroblasts. The DLS results demonstrated that the mean hydrodynamic diameter of particles increased from $25 \mathrm{~nm}$ to $183 \mathrm{~nm}$ [2]. The analysis of Au NPs, synthesized in citrate media as 
reducing agent, by DLS revealed that the surfaces of the NPs were covered by citrate. As it has been mentioned above, proteins and other biological molecules may bind to the surface of the MNPs. The absorption spectra of Au NPs in solution showed that the NPs were not only covered by citrate, but also proteins or lipids were adsorbed onto their surface [37].

\subsection{Localization of MNPs into cells and living organisms using microscopic techniques}

TEM is also considered an appropriate technique to discriminate between MNPs localized inside cells and tissues from those that are only externally attached. Obtaining reliable information about the intracellular localization of MNPs is important to establish NP-cell interactions. It is known that smaller NPs can enter more easily into cells than larger ones [38], which might explain the higher toxicity of smaller MNPs. When it come to design a protocol for electron microscopy, it is very important to select the most suitable fixing agent. The most common fixings are aldehydes (glutaraldehyde and formaldehyde) and osmium tetroxide. Aldehydes react with amines and amino acids (lysine and arginine), generating cross-linked proteins, which preserve cellular structure. However, these fixing agents do not react with most lipids of the cellular membrane leading to problems during fixing and processing. For this reason, osmium tetroxide is generally used as a secondary fixing since it is able to reacts with unsaturated lipids and with some proteins, thus stabilizing the membrane structures and also adding electron density to enhance the contrast. Another important aspect is the choice of buffer in which the fixing will be dissolved. It should maintain the cell in its physiological state when possible. The most frequently used buffer is PBS. Buffers containing amine groups, such as Tris, should not be used because these groups might react with the fixing molecule. Typically, samples prepared for TEM analysis have to be dehydrated and embedded in resin (overnight with temperature) to allow the cut of ultrathin sections. Allouni et al. developed a protocol in which cells were fixed in $1.5 \%$ glutaraldehyde and $0.1 \mathrm{M} \mathrm{Na-cacodylate} \mathrm{buffer} \mathrm{(pH} \mathrm{7.4),} \mathrm{and}$ post fixed in $1 \%$ osmium tetraoxide. TEM images revealed that $\mathrm{TiO}_{2} \mathrm{NPs}$ were not only attached to the surface of the cells, but also they were in vesicles and distributed in the cytoplasm [11]. A mixture of glutaraldehyde and formaldehyde is also very commonly used as fixing agent. A fixing solution containing 3\% paraformaldehyde and $0.5 \%$ glutaraldehyde was employed to visualize both the localization of $\mathrm{SiO}_{2} \mathrm{NPs}$ and $\mathrm{Ag}$ doped $\mathrm{SiO}_{2} \mathrm{NPs}$ in fibroblast-like fathead minnow cells [17], and $\mathrm{Au}$ NPs in human lung carcinoma cells A549 [37]. In some cases, resulting blocks embedded in resin are incubated in uranyl-acetate $(1 \%-6 \%)$ to obtain a higher electron density and image contrast, which is especially useful for smaller particulate specimens. However, this stain is not recommended in the treatment of specimens that are unstable in acid conditions due to the low $\mathrm{pH}$ of solution $[7,11]$. Ultrahigh resolution microscopy (URI) is a promising technique for studying cells-NPs interactions as it allows direct visualization of both cells and MNPs without the need of an elaborate sample preparation and without the use of fluorescent tags. In URI the illuminator is connected to the light source by a liquid light guide, which allows transport of light without loss of intensity and minimizes the thermal impact on living systems. In a recent study, the cellular uptake of $\mathrm{TiO}_{2} \mathrm{NPs}$ by fibroblasts was investigated by combining scanning (SEM) and transmission (TEM) electron microscopy with URI. For URI analysis, cells were washed, fixed in $4 \%$ formaldehyde for $15 \mathrm{~min}$ at room temperature and finally, a drop of mounting media was added to the slides and encased with a cover-slip. URI images showed that $\mathrm{TiO}_{2}$ NPs appeared to be both up-taken by and attached to the cells, mainly as agglomerates. The 
discrimination between MNPs attached to the cell from those internalized was performed by changing the focus plane in the URI microscope. As shown in Fig. 1, the agglomerates could be easily distinguished from other cellular components due to their brightness [11].

Dark-field microscopy (DFM) is able to provide a panoramic view of non-fluorescent NPs in cells since NPs appear as a bright image super imposed on a dark background. Because dark-field illumination has a very low background, low signals are sufficient to detect individual non-fluorescent NPs. Although there are limited studies on non-fluorescent NPs accumulation at the single-cell level using DFM, this technique has been used in studies focused on Au NPs. As in URI, sample preparation is very straightforward when using dark field imaging. The accumulation of Au NPs in cells has been observed in a time-course experiment. Cells were seeded on coverslips in 24-well plate and fixed in 4\% paraformaldehyde for 10 minutes after MNPs exposure [26], and Au NPs embedded inside the cell were identified in their aggregated states [17].

Another alternative for visualizing MNPs is based on its functionalization with fluorescent markers, which allow for using fluorescence microscopy. However, it is important to keep in mind that the attachment of a fluorochrome may lead to the alteration of the physicochemical properties of MNPs. MNPs can be easily functionalized by using specific fluorochromes, which have their own absorption and emission spectra. Fluoresceien isothiocyanate (FITC), ALEXA 488, BODIPY- FL, propidium iodide, Mito-Tracker Red and rhodamine, among others, are some of the fluorochromes commonly used in many bioapplications. FITC presents maxima absorption in the blue light range of the visible light spectrum (approximately at $488 \mathrm{~nm}$ ). When FITC is irradiated at $488 \mathrm{~nm}$, it fluoresces at $520 \mathrm{~nm}$, emitting a greenish-yellow glow. Other fluorochromes such as propidium iodide, ALEXA 488 and BODIPY- FL have excitation and emission profiles similar to FITC. On the contrary, rhodamine 123 and Mito-Tracker Red fluorochrome are excited by light in the green range and emit in the red wavelengths. Kurepa and coworkers synthesized Alizarin red S (ARS)- $\mathrm{TiO}_{2}$ NPs conjugates to study the NPs uptake and distribution in plants. The covalent surface modification of the MNPs was performed following a straightforward protocol in which the MNPs were mixed with an ARS solution. The high surface reactivity of synthesized $\mathrm{TiO}_{2} \mathrm{NPs}$ (smaller than $20 \mathrm{~nm}$ ) was very adequate in the formation of ARS-NPs conjugates [38].

\section{In vitro cytotoxicity assays}

Cytotoxicity assays are based on the idea that toxic agents affect basic functions of cells, and thus, their toxicity can be evaluated by measuring cellular damage. It is known that the toxic effects of MNPs on a cell culture may be different depending on the incubation time and the concentration of MNPs used. Many cytotoxic effects are due to the number of internalized MNPs into the cells rather than to the total concentration of NPs added to the cell culture media. Generally, the amount of MNPs uptaked by the cells differs in several orders of magnitude from the amount of NPs used in the cellular treatment [39]. The uptake efficiency does not only depend on the MNPs concentration but also on other properties such as MNPs size and shape. Exposure times and concentrations greatly vary from one study to another. Concentrations in the order of $\mu \mathrm{g} / \mathrm{L}$ or a few $\mathrm{mg} / \mathrm{L}$ are commonly used, while exposure times ranging from 24-96 hours are usually selected [6, 9, 40], although internalization of MNPs has been observed at shorter exposure times under certain conditions $[15,41]$. 
When performing a cytotoxicity assay, cell types are generally chosen considering the organ in which the studied MNPs are accumulated in organisms. In this term, hepatic cells [42, 43] and macrophages [2] are the most commonly used since MNPs tend to accumulate in liver and spleen. Other cell types such as fibroblasts, which are the major cellular constituent of fibrous connective tissue, have also been used for example to assess the effects of $\mathrm{TiO}_{2} \mathrm{NPs}$ released from implant materials [11]. It is important to keep in mind that the toxic effects induced by MNPs might depend on the cell line tested and these effects may not necessarily have to be the same when exposing other cell lines to the same MNPs $[44,5]$. In this way, Patra et al. found that citrate-capped Au NPs induced a higher toxicity in human carcinoma lung cells as compared to liver carcinoma cells at the same dosage [45]. Actually, the effects could even be the opposite as shown by Chen et al., where Se NPs inhibits cellular growth in hepatocarcinoma cells but increases cell proliferation when using human fibroblasts [44].

Below, we describe several common assays that have been applied when evaluating the effect of MNPs in different cell lines, most of them involving the use of vital dyes, protease markers, and colorimetric, fluorescence, or luminescence detection.

\subsection{Assessment of membrane integrity}

The cell membrane is a thin semi-permeable membrane that surrounds the cytoplasm of a cell and constitutes a functional barrier. Its function is to protect the integrity of the cell by controlling the traffic into and out of the cell, which is regulated by transporters, receptors and secretion pathways. Since cytotoxic compounds often compromise the cell membrane, assessing cell membrane integrity is one of the most common ways to measure cell viability and cytotoxic effects. For this purpose, the so-called vital dyes, such as trypan blue and propidium iodide, are generally used, which are compounds able to freely cross the cell membrane when it has been compromised and stain intracellular components, but are excluded from the inside of healthy cells [46]. Alternatively, membrane integrity can be evaluated by monitoring the presence in the culture media of substances that are normally sequestered inside healthy cells [5]. One commonly measured is lactate dehydrogenase (LDH) [47]. LDH leakage from damaged cells can be measured with a coupled enzymatic assay resulting in the conversion of a tetrazolium salt into a red formazan product. The intensity of the color produced is proportional to the number of cells with compromised plasma membrane [37]. There are several commercially available kits to carry out the this assay, which does not involve any kind of sample preparation since the substrate mixture is directly added to the culture media and the resulting reaction product (red formazan) is measured spectrophotometrically. Using this assay, Hussain et al. showed that $\mathrm{LDH}$ leakage for $\mathrm{MoO}_{3}, \mathrm{Al}, \mathrm{Fe}_{3} \mathrm{O}_{4}$, $\mathrm{MnO}_{2}$, and W NPs did not produce cytotoxicity up to a concentration of $100 \mu \mathrm{g} / \mathrm{mL}$ in rat liver cells, while Ag NPs were highly toxic at those concentrations in the same cells [10]. In the same manner, Gerloff et al. showed that only anatase-rutile containing NPs, in contrast to the pure fine $\mathrm{TiO}_{2}$, induced cytotoxicity in human intestinal cells [3].

\subsection{Measurement of cellular metabolic activity}

An early indication of cellular damage is a reduction in metabolic activity. Thus, assays able to measure metabolic functions such as ATP levels or mitochondrial activity have been widely used to evaluate the toxicity of metallic nanoparticles. One of the most widely used methods is the 3-(4, 5-Dimethyl-2- 
thiazolyl)-2, 5-diphenyl-2H-tetrazolium bromide (MTT) assay [14, 48], which measures the reducing potential of a cell. It is based on the capacity of healthy cells to reduce MTT to formazan by mitochondrial reductases. Formazan is a colored compound whose absorbance can be measured spectrophotometrically at a wavelength between 500-600 nm. In this approach, cells are incubated with MTT (2-10 mg/L) between 4-5 hours and then the formazan crystals are commonly dissolved in dimethyl sulfoxide (DMSO) (or other organic solvent) prior colorimetric detection. It is important to consider the signal provided by the phenol red usually present in culture media. Thus, it is recommended to either use red-phenol free media or to remove the media before dissolving the formazan crystals. The cytotoxicity of several MNPs has been evaluated by comparing the absorbance of formazan in control vs. cells exposed to MNPs. Christen and Fent showed that human hepatic cells (Huh7) reacted more sensitively to $\mathrm{SiO}_{2}$ NPs than fibrolast-like fish cells (FHM). Exposure of the two cell lines with $\mathrm{SiO}_{2}-\mathrm{Ag} \mathrm{NPs}$, containing 1, 5 and $20 \%$ of Ag demonstrated again that Huh7 cells were more sensitive than FHM cells (Fig. 2) [7]. Yuan et al. used the MTT assay to show how the viability of lung fibroblast exposed to $\mathrm{ZnO}$ decreased in a dose-dependent manner independently of the particle size [49]. Other close dyes such as 3-(4,5dimethylthiazol-2-yl)-5-(3-carboxymethoxyphenyl)-2-(4-sulfophenyl)-2H-tetrazolium (MTS), 2,3-bis-(2methoxy-4-nitro-5-sulfophenyl)-2H-tetrazolium-5-carboxanilide (XTT) and water soluble tetrazolium salts (WSTs) have also been used for the same purpose $[3,6]$.

\subsection{Evaluation of the oxidative stress}

Oxidative stress is an imbalance of pro-oxidants and antioxidants. It is associated with increased production of oxidizing species or a significant decrease in the effectiveness of antioxidant defenses. One common way to analyze oxidative stress in cells is by measuring glutathione (GSH) levels. GSH is the major intracellular low-molecular-weight thiol that plays a critical role in cellular defense against oxidative stress. There are many commercially available GSH detection kits mainly based on colorimetric and fluorimetric detection. One key step in this approach is the thorough wash of the cells with PBS to remove the excess of dye prior detection. GSH measurement is widely used in toxicological studies [50] and it has also been used to evaluate the toxicity of different MNPs. For example, GSH depletion has been observed in cells exposed to Ag NPs, although the degree of depletion greatly depended on the cell line tested [5]. When cells are exposed to environmental stress they can generate reactive oxygen species (ROS), both radical ROS (nitric acid and hydroxide radicals) and non-radical ROS (hydrogen peroxide). When the increase of ROS is too high and it overwhelms the antioxidant defense, cells are prone to suffer cell damage and even apoptosis. Several studies have linked the increase of the ROS level in cells with the exposure to MNPs; thus allowing the use of the ROS level as a cytotoxicity marker. The most widely used method to measure the intracellular production of ROS is the dichlorodihydrofluorescein diacetate (DCFH-DA) assay [8]. DCFH-DA penetrates the cells, where it is hydrolyzed by unspecific esterases and converted into a stable green fluorescent product (DCF) in the presence of oxidative species [53]. DCFHDA is prepared in phenol red-free media and used at a final concentration of $10 \mu \mathrm{M}$. Cells are incubated with this reagent for 15-30 min and then washed twice with phosphate buffer saline (PBS) prior fluorimetric detection. It is important to carry out this assay in the dark to avoid fading of the fluorophore. Using this approach, Konczol et al. demonstrated that the ROS production observed in alveolar epithelial cells increased after $24 \mathrm{~h}$ of incubation with $\mathrm{Fe}_{3} \mathrm{O}_{4} \mathrm{NPs}$ in a concentration-dependent manner. This study 
also showed that the particle size was not related with the cytotoxicity of these MNPs [51]. Reaction of generated ROS with other fluorescent probes such as dihydroethidium (DHE) has also been used [52]. In addition, when the ROS production increases, other intracellular events like lipid peroxidation are initiated; thus, by measuring these events it is also possible to evaluate the oxidative damage of cells exposed to MNPs. Malondialdehyde (MDA) formed from the breakdown of polyunsaturated fatty acids, can be used as a convenient index for determining the extent of lipid peroxidation reactions. MDA formation can be measured by western blot [53], enzyme-linked immunosorbent assay (ELISA) or by the traditional thiobarbituric acid reactive substances (TBARS) assay based on the reaction of MDA with thiobarbituric acid reactive susbtances to yield a fluorescent product [41]. After cell lysis, the cell extract is mixed with the reaction solution and incubated at $90^{\circ} \mathrm{C}$ for $1 \mathrm{~h}$. The product formed is extracted with an organic solvent such as butanol and the fluorescence is measured in the organic phase. Addition of cadmium chloride to control cells can be used as positive control since it induces lipid peroxidation [52]. Passagne et al. used this assay to show how $\mathrm{SiO}_{2}$ NPs affected the production of MDA depending on the cell line tested and the particle size. While smaller NPs $(20 \mathrm{~nm})$ induced the formation of MDA in porcine tubular cells to a larger extent as compared to bigger NPs $(100 \mathrm{~nm})$, the effect of both NPs was not significantly different when using human kidney cells [52].

\subsection{Measuring apoptosis induced by MNPS}

As commented before, exposure of cells to MNPs can increase ROS production and lipid peroxidation, which ultimately can cause apoptosis. There are several strategies to evaluate the degree of apoptosis based on the measurement of different parameters. There are several protein markers of apoptosis including Bax, Bc12, p53 and caspases that can be easily monitorized using Western Blot with the appropriate antibodies $[8,55]$, colorimetric assays [56] or real-time polymerase chain reaction (PCR) [38, 58]. Apoptosis can also be visualized by studying nuclear morphology, particularly chromosome condensation, assessed using nuclear specific dyes such as 4'-6-diamidino-2-phenylindole (DAPI) and Hoechst and fluorescence microscopy $[54,55]$. However, the general chosen technique to evaluate apoptosis is flow cytometry. The most important factor that have to be considered when preparing samples for flow cytometry is the density of the cell suspension, which should be kept between $10^{5}-10^{7}$ cells/mL to prevent the narrow bores of the cytometer and its tubing from clogging. Two common methods to evaluate apoptosis by flow cytometry are the measurement of the mitochondrial membrane potential and the annexin-V assay. Since mitochondria act as a point of integration for apoptotic signals originating from both, the extrinsic and the intrinsic apoptotic pathways [57]; loss of mitochondrial membrane potential can be associated with the activation of caspases and the initiation of apoptotic cascades [58]. To measure the percentage of cells with depolarized mitochondria, resuspended cells are incubated at $37^{\circ} \mathrm{C}$ for $10-15 \mathrm{~min}$ with a solution of $10 \mu \mathrm{g} / \mathrm{mL} \mathrm{JC}-1$ (5,5',6,6 '-tetrachloro-1,1',3,3'tetraethylbenzimidazolcabocyanine iodide). After incubation, cells are immediately centrifuged to remove the supernantant and the cell pellet is resuspended in PBS and then analyzed by flow cytometry. The membrane-permeant JC-1 dye exhibits potential-dependent accumulation in mitochondria, indicated by a fluorescence emission shift from green $(\sim 529 \mathrm{~nm})$ to red $(\sim 590 \mathrm{~nm})$. Consequently, mitochondrial depolarization is indicated by a decrease in the red/green fluorescence intensity ratio. The potentialsensitive color shift is due to concentration dependent formation of red fluorescent J-aggregates [54]. 
Using this method, Chen et al. demonstrated that SeNPs induced apoptosis in melanoma cells through mitochondrial-mediated pathways. In this study, de percentage of cells with depolarized mitochondria increased from $5.6 \%$ (control) to $9.3 \%$ when exposing the cells to $20 \mu \mathrm{M}$ SeNPs, and up to $30.0 \%$ when using $40 \mu \mathrm{M}$ SeNPs [56].

In apoptotic cells, phosphatidyl serine (PS) is translocated from the inner to the outer leaflet of the plasma membrane; thus, PS becomes exposed to the external cellular environment. Annexin V labeled with a fluorophore such as FITC, can selectively identify apoptotic cells by binding to the exposed PS on the outer leaflet of the membrane. The number of labeled cells can then be quantified by flow cytometry. Annexin V-FITC is usually used together with propidium iodide (PI) to differentiate between apoptotic and death cells. Cells are resuspended $\left(10^{6} \mathrm{cells} / \mathrm{mL}\right)$ in binding buffer $(10 \mathrm{mM}$ HEPES/NaOH, $\mathrm{pH} 7.5$ containing $140 \mathrm{mM} \mathrm{NaCl}$ and $2.5 \mathrm{mM} \mathrm{CaCl}_{2}$ ) and then incubated in the dark with annexin V-FITC and PI for $10 \mathrm{~min}$ at room temperature [54]. Generally, fluorescence emitted by annexin V-FITC and DNAbound PI in each event is detected as green and red fluorescence, respectively. Other fluorophores can also be conjugated to annexin- $\mathrm{V}$ [44]. This assay is generally used to confirm that the cell death observed after exposure to certain MNPs is generally caused by apoptosis [44, 59]. It is important to mention that certain fluorochromes may be adsorbed on the surface of MNPs, which may cause fluorescence of the NPs, and quenching or enhancement of the fluorescence of fluorochromes, thus altering the results of the flow cytometry assay. In addition, cellular internalization or deposition of MNPs may avoid the correct binding of the fluorochromes to their target molecules. As an example, the annexin- $\mathrm{V}$ assay may gives false negatives as a result of NPs deposition on the cellular membrane, which hinder the annexin-V from binding to PS.

\subsection{Genotoxicity assesment}

The potential DNA damaging properties of MNPs has also been assessed in many cases using the Comet and the formamidopyrimidine glycosylase (Fpg)-modified Comet assay [3, 51, 54]. The comet assay (also known as single cell gel electrophoresis) is a rapid, sensitive and simple method for detecting DNA damage at the level of individual cells [60]. It involves encapsulation of single cells in low melting point agarose. Then, cells are lysed to form nucleoids containing supercoiled loops of DNA. Electrophoresis at high $\mathrm{pH}$ results in DNA structures resembling comets. It is important to reduce the $\mathrm{pH}$ after electrophoresis to avoid the contribution of alkali-labile sites since they strongly decrease the sensitivity of the comet assay. The extent of DNA migration depends directly on the DNA damage present in the cells. It should be noted that DNA lesions consisting of strand breaks after treatment with alkali either alone or in combination with certain enzymes (endonucleases) increases DNA migration, whereas DNADNA and DNA-protein cross-links result in retarded DNA migration. This is followed by visual analysis with staining of DNA with ethidium bromide [54,61]. Alternative dyes such as propidium iodide, Hoechst or DAPI have also been used. The intensity of the comet tail relative to the head reflects the number of DNA breaks. Gong et al. used this assay to evaluate the effect of $\mathrm{SiO}_{2} \mathrm{NPs}$ of different particle size in human keratinocytes showing a significant increase in the comet tail length of cells exposed to smaller $\mathrm{SiO}_{2}(15 \mathrm{~nm})$, which means an increased DNA damage in a NP size-dependent manner (Fig. 3) 
[62]. Several studies used a modification of the comet assay in order to specifically evaluate oxidative DNA damage. For this purpose, the endonuclease formamido pyrimidine glycosylase (Fpg) is generally used for detection of 8-OH guanine, other damaged purines, basic sites (AP sites) and ring-opened N-7 guanine adducts [63]. This endonuclease is added to the gel and incubated for $30 \mathrm{~min}$ at $37^{\circ} \mathrm{C}$ in a humidified chamber. It is important to prepare this enzyme in a buffer solution containing bmercaptoethanol to preserve the enzyme and to take into consideration that the inclusion of sulphydryl reagents in the reaction buffer would significantly increase background DNA breakage. Gerloff et al. demonstrated that only anatase/rutile $\mathrm{TiO}_{2} \mathrm{NPs}$ significantly increased DNA strand breakage while none of the anatase $\mathrm{TiO}_{2} \mathrm{NPs}$ induced oxidative DNA damage [3].

\subsection{Use of microscopy to study cellular morphological alterations}

Several studies derived from electron microscopy analyses have reported morphological alterations in the cell layer and/or cell shape after exposure to cytotoxic agents. Therefore, it is not surprising that the visualization of morphological alterations is considered as a primary index of toxicity. Changes in the morphology of lung fibroblasts were examined by SEM after incubation with $20 \mathrm{~nm}$ ZnO NPs for 72 hours. For this analysis, cells were washed with PBS and dehydrated in 35\% alcohol for $10 \mathrm{~min}$, followed by consecutive addition of ethanol solutions from $40 \%$ to $100 \%$. Finally, the samples were dried in vacuum before SEM analysis. A very rough cell surface was observed in ZnO NPs treated cells as compared with control cells, which have a regular morphology and smooth surface. Moreover, the presence of nanosized pores of $20 \mathrm{~nm}$ in the plasma membrane indicated that ZnO NPs could have entered into the cells, thus affecting the inner structure and inducing apoptosis and/or necrosis [49]. Unlike SEM or TEM techniques, the use of phase contrast microscopy allowed examining living cells in its natural state without the need of previous fixation or dehydration steps. Hussain et al. observed that increasing the Ag NPs concentration in culture media led to morphological changes in rat liver cells, which started to shrink and became irregular in shape [10]. In the same way, the cellular morphology of epithelial carcinoma cells was observed in order to evaluate the cytotoxicity of $\mathrm{Fe}_{3} \mathrm{O}_{4} \mathrm{NPs}$ with different surface functionalizations [14]. For a more exhaustive visualization of the morphological changes, cells may be fixed and differentially using the hematoxylin-eosin method. Whereas hematoxylin (blue-purple dye) stains nucleic acid, eosin (pink dye) stains proteins nonspecifically. The required protocol for sample preparation is very straightforward. In a study of cytotoxicity of Fe NPs, HeLa cells were seeded on the glass coverslips in 24-well plate and incubated for $2 \mathrm{~h}$ with the NPs. Then, cells were fixed with $4 \%$ paraformaldehyde and stain with hematoxylin and eosin. In this case, no significant changes in cellular morphology was observed after exposure to Fe NPs [30], which could be due to the extremely short exposure time selected to carry out the study.

\subsection{High throughput discovery platforms}

In addition to the assays commented before, which are mainly based on target-directed approaches, high throughput discovery platforms based on gene and protein expression profiling can provide very useful information on the specific mechanisms of interaction of MNPs with cells. Gene expression is the term used to describe the transcription of the information contained within the DNA into messenger RNA (mRNA) molecules that are then translated into proteins. The study of the kinds and amounts of mRNA 
produced by a cell under certain conditions, which is directly related to which genes are expressed, provides insights into how the cell responds to a particular perturbation. A way to evaluate the expression of many genes in a single experiment is by using DNA microarrays. A DNA microarray is a collection of microscopic DNA spots immobilized in a solid surface. Each DNA spot contains picomoles of a specific DNA sequence, known as reporters (also known as probes or oligos), which can be a short section of a gene or other DNA element used to hybridize a cDNA or a cRNA. Probe-target hybridization is usually detected and quantified by detection of fluorophore, silver-, or chemiluminescence-labeled targets to determine relative abundance of nucleic acid sequences in the target. Hanagata et al [64]. carried out a whole genome expression analysis to study the molecular responses of lung epithelial cells to CuO NPs. After extraction of the RNA from cells exposed to $\mathrm{CuO}$ (or control cells), the RNAs were amplified and labeled with fluorescence probes (Cy3 and Cy5). Hybridization of the amplified RNAs with the DNA microarray was carried out for $18 \mathrm{~h}$ at $65^{\circ} \mathrm{C}$. Then, the microarray was scanned to measure the fluorescence intensity of $\mathrm{Cy} 3$ and $\mathrm{Cy} 5$. The foreground and background median values of each spot were obtained from median values of the pixels included in each spot area. The difference between the foreground and background values of each spot was established as the signal strength, and only spots with signal values $>3$ times the noise value were considered valid spots. The results indicated that change of gene expression involved in mitogen-activated protein kinases (MAPKs) pathways and cell cycle progression in cells exposed to $\mathrm{CuO}$ NPs was similar to that in cells exposed to $\mathrm{Cu}$ ions [64]. With a similar approach Mahmoudi et al. evaluated the toxicity of superparamagnetic iron oxide in different cell lines (heart, brain and kidney) [65] and Dua et al. the toxicity indeced by mesoporous silica- $\mathrm{NPs}_{2} \mathrm{Fe}_{2} \mathrm{O}_{3}$ NPs and ZnO-NPs in kidney cells [66]. The findings using this approach have to be analyzed by means of powerful bioinformatic tools due to the large amount of data obtained. In addition, alternative methods such as real time-PCR are also required for further validation of the identified targets. Examining changes in protein expression can also be very useful for better understanding the effects of MNPs in cells [67].

Among all the proteomic approaches available, quantitative strategies are particularly attractive since they allow for the identification of differentially expressed proteins in cells exposed to MNPs as compared to control cells. Although these approaches are extensively being used in other research areas [68], they have been applied to study MNPs toxicity in only a few studies. Yang et al. used twodimensional differential gel electrophoresis (2D-DIGE) to identify proteins altered after $\mathrm{SiO}_{2}$-NPs exposure. In this study, control and cells exposed to $\mathrm{SiO}_{2}$ were differentially labeled with $\mathrm{Cy} 3$ and Cy5 dyes for comparison on the same gel. The labeling reaction was carried out for $30 \mathrm{~min}$ at $4^{\circ} \mathrm{C}$ in the dark and then quenched with a 50 fold molar excess of free lysine. The internal standard was labeled with Cy2 dye and use on all gels to aid image matching and cross-gel statistical analysis. The proteomic analysis revealed that 16 differentially expressed proteins were induced by $\mathrm{SiO}_{2} \mathrm{NPs}$ exposure, and that the expression levels of these proteins were associated with the particle size [69]. Jeon et al. also used 2DDIGE (two-dimensional difference gel electrophoresis) to analyze the hepatotoxicity induced by $\mathrm{TiO}_{2}$ in liver cells, finding 15 altered proteins related to inflammation, apoptosis and antioxidative reaction [70]. Alternatives to 2D-DIGE, such as stable isotopic labeling by amino acids in cell culture (SILAC), isobaric tags for relative and absolute quantitation (iTRAQ) and label-free methods should also be 
considered for future studies since they are generally providing better results than gel-based approaches [67].

\section{In-vivo assessment of metallic nanoparticles toxicity}

After in vitro toxicity assays and before the pre-clinical studies, in vivo assays aim to evaluate the toxicity of MNPs are needed to design specific and safe clinical trials to develop new strategies for the applied use of MNPs. The use of this compounds include diagnostic and/or therapeutic purposes although large-scale use of MNPs also represent an increasing risk to the health and environment $[18,71]$. Several aspects of MNPs physicochemical properties are important to design these protocols and of course, the suitable biological organism in each case. The target organ in the organism chosen is also one of the major issues to test. Therefore it is important to test hepatotoxicity, nephrotoxicity, pulmonary toxicity, spleen toxicity, immune system activation, hematological toxicity and oxidative stress among others [72, 73]. Within the most important options to consider is the route of NPs administration, especially in higher vertebrates and mammalian.

\subsection{Routes of administration in common used animal models}

The acquisition pathway of MNPs by biological systems is determinant, in most cases, to the type and extent of potential damage resulting in different organs and tissues. Relevant routes of administration and doses in different animal model are discussed below.

4.1.1. Skin absorption. This route of administration involves not only the active absorption after direct application to the skin but also passive uptake of NPs through the skin as, for example, by respiration after nebulization in a chamber or directly in the water, for aquatic animals [74]. The zebrafish is a genetically-tractable vertebrate model organism commonly used for the in vivo administration and testing of MNPs toxicity. Compared to other vertebrate animal models, zebrafish have several benefits. Importantly, the housing and maintenance is less expensive what makes it an attractive model for preliminary studies of toxicity and/or environmental contamination. Moreover zebrafish model for the assessment of toxicity are easily extrapolated to other vertebrates and humans. The short life cycle of this aquatic vertebrate makes this model very suitable for the study of embryo viability and genetic malformations during the treatment with NPs. Assessment of MNPs toxicity in zebrafish includes immune-hystochemical analysis, flow cytometry studies to detect reactive species of oxygen and proteomic analysis for well-established stress cellular markers [75]. Eurasian perch, oysters and the fruifly share many of the zebrafish characterisitics to make them suitable models for toxicity studies although are used less frequently because their genetic is not well known.

4.1.2. Airways release. Ag and Au NPs in vivo toxicity have been studied in models of sub-chronic inhalation trying to have a complete study of toxicity that measure the adsortion, distribution, metabolism and excretion (ADME) of the MNPs in vivo in rats [76]. These studies, although not very common, need to be done for commercial purposes and for the use of MNPs in humans. The most important parameters are the minimum dose for an adverse effect (LOAEL) and the dose without an adverse toxicity effect included in the pre-clinical trial (NOAEL). These studies tested daily different doses of, for example, Au 
NPs in different groups of male and female rats with 6 hours inhalation treatment consecutively during three months. Airways, skin and mucosas (oral, nasal and genital) were checked daily and the rats weight was monitored once in a week. The analysis before mortem included biochemistry and hematologic parameters, nephrotoxicity and lung function. The post-mortem study consisted in multiorganic histopathologycal analysis (including broncho-alveolar lavage). Au accumulation in tissue was measured by atomic absorption spectrophotometer [76].

\subsubsection{Systemic administration. Commonly used routes for systemic release of MNPs are intra-venous} (i.v.) and intra-peritoneal (i.p.) injection. The systemic administration of MNPs by these two pathways allows the study of their organ distribution after different time points. The organs are extracted from injected mice and accumulation of nanoparticles is generally analyzed by ICP-MS and/or by fluorescent imaging with confocal microscopy. Interestingly, silica-overcoated magnetic small size (50 nm) nanoparticles containing rhodamine B isothiocyanate were recruited to the brain, through the blood brain barrier [77], whereas Au NPs with a diameter of 100-250 nm were retained in different tissues and organs such as liver, spleen, kidney, or lungs [78]. Hematological and biochemical studies are also performed in these animals to test the toxicity of nanoparticles in tissues and blood cells. Other interesting approach with systemic administration of nanoparticles is the evaluation of mutagenic potential, chromosomal aberrations and genotoxicity for germinal cell viability studies [77, 79].

4.1.4. Oral gavage. This route of administration has been studied using $\mathrm{Cu}$ NPs from different sizes, after gavage of mice through naso-gastric probe. In this model of acute toxicity, the effect of the NPs was tested after short exposure to high doses. After determination of the $\mathrm{LD}_{50}$ (lethal doses determination for $50 \%$ of the population), a complete histopathology and hematology study has to be performed to describe the ADME parameters in vivo in the animal model.

Oral toxicity determination is important due to the putative use of nanoparticles with food or oral administrated drugs $[80,81]$. Moreover, oral uptake of nanoparticles through drinking water pollutants or passive uptake from other routes of administration as airways or skin, must been taken into consideration to fully address the in vivo toxicity of MNPs [82]. A single administration of NPs is performed in the acute exposition model for MNPs toxicity assessment; hence increasing doses of the different NPs to test and the control (vehicle) are administrated to the mice to detect the $\mathrm{LD}_{50}$. In an interesting study, Chen, et al. tested two sizes of $\mathrm{Cu}$ NPs $(25 \mathrm{~nm}$ and $17 \mathrm{~nm})$, and compared the effect to that of soluble ionic $\mathrm{Cu}$ after nasogastric probe administration. This study revealed the importance of the size of the NPs for the induction of toxicity; larger NPs have small amount of surface in contact with the organism and are, therefore potentially less toxic than the smaller nanoparticles or the ionic $\mathrm{Cu}$. Other important conclusion from this work is that the study of the different ways of nanoparticles administration is essential to understand the differential and specificity of MNPs toxicity induction. As an example, the interaction of NPs with $\mathrm{H}^{+}$in the stomach after oral administration promotes an increase in the release of $\mathrm{HCO}^{-}$, which results in an increase of the $\mathrm{pH}$ in the stomach causing renal damage and electrolytic metabolic alterations [82].

Another important issue after oral administration of NPs is the different outcome depending on the use of males or females for toxicity tests. In this regard, males are more prone to produce $\mathrm{H}^{+}$in the 
stomach and therefore they are more susceptible to develop renal damage as compared to females [83]. All these considerations remark the importance for designing an adequate in vivo toxicity experimental model. Moreover, a deep knowledge of the biology from the selected animal is needed to properly assess the extent of the route of administration in the toxicity induced by MNPs [82].

\subsection{Alternative in vivo models}

Other organisms as invertebrates, which permits the analysis of ADME toxicity parameters, have also been used for the in vivo evaluation of MNPs toxicity. An important benefit of these models is the avoidance of bioethical committees.

\subsubsection{Invertebrate organisms. In an interesting study, Novak and collaborators proposed a terrestrial} invertebrate, Porcellio scaber (Isopoda, Crustacea) to test toxicity of $\mathrm{TiO}_{2} \mathrm{NPs}$ orally administrated [84]. They hypothesized that the $\mathrm{TiO}_{2}$ nanoparticle toxicity could be due to the high doses, exposition time and the ability to distribute within the whole animal body after crossing gut epithelial cells barriers. The route of administration by feeding the animal with NPs within the intake food permitted the study of several parameters as general health score of the animals. Each individual was independently exposed and the feed rate was calculated by the relation between food consumption and weight increase from each animal and each day of the treatment. The spreading ability of NPs to the whole organism was evaluated by analyzing the membrane integrity of gut epithelial cells by immunohistochemistry [84, 85].

The use of environmental contamination biomarker organisms is other interesting approach as a model to test MNPs induced toxicity. In this regard, the freshwater crustacean Daphnia magna, has been used as a contamination biomarker due to its sensitivity to changes in the ecosystem [86]. Moreover, Daphnia magna, is part of the trophic chain base revealing important information about the accumulation of toxic effects within the rest of the organisms in the ecosystem [87, 88]. In a recent work Asghari et al. evaluated the toxicity of different groups of colloidal Ag NPs under an acute and short-term exposition model. They determined the minimum dose of Ag NPs inducing lethality in D. magna after 48 hours administration in water containers. The toxicity score was evaluated by lethal doses determination $\left(\mathrm{LD}_{10}\right.$, $\mathrm{LD}_{50}$ and $\mathrm{LD}_{90}$ ), observation of natation defects and progression of paralysis. They concluded from these studies that the toxicity of Ag NPs depends on their nature and the aggregation state [89]. Similar studies have been performed by other groups to analyze the effects of silver nanoparticles ingested by $D$. magna $[89,90]$.

It is interesting to remark a number of benefits and disadvantages derived from the use of invertebrate models to test toxicity induced by MNPs. First, the protocols are technically easier as compared to vertebrates and mammals and therefore simplified read-out interpretation. These animals are big enough to obtain such amounts of biological samples for histopathologic analysis and other purposes. Other important issue is the possibility to obtain high number of individuals to increase statistical significances. Conversely, nowadays the short experience in these models could be a disadvantage to their use as MNPs testers. Other objection to the use of invertebrates is the lack of mathematical models to properly read into the results and to extrapolate to the potential toxicity in humans. The last concern could be aggravated due to the low physiological correlation between vertebrates and invertebrates. Hence, the 
ideal situation for properly test the toxicity of MNPs, would be the use of both types of model animals, invertebrates and vertebrates.

4.2.2. Embryo models. Other important factor to be considered is the potential toxicity of MNPs during embryonic development. To address this question, Clancy et al. have developed a model of live chicken embryo in which MNPs are administered directly in the blood flow. The use of fluorescent NPs, allowed the study of their distribution through the whole body, as well as the aggregation behavior, by fluorescence spectroscopy. An interesting observation is that NPs tended to aggregate and accumulate in blood vessels from different sizes and in tissues with high angiogenic activity. Similarly to the toxicity studies in vertebrates, the toxicity in embryo models depends on the bio-distribution of the MNPs and their physicochemical properties: size, electrical charge, surface modifications (chitosan, fluorescent particles, etc.) [91, 92].

The data obtained from living chicken embryo could be extrapolated to adult animals being helpful for the development of toxicity protocols. Moreover, chicken embryo is a bonafide model to set up this experimentation as their use is not regulated by ethical committees, thus avoiding legal issues [92].

4.2.3. Procariotic organisms. A new biological model using procariotic organisms has been recently proposed for MNPs toxicity studies. This model is based on the use of bacteria able to produce bioluminescence. Bioluminescence intensity is directly related to cellular metabolism and this correlation is used to address the toxicity of the NPs. A decrease in metabolic activity induced by MNPs exposure, would result in a decrease in the bioluminescence intensity, which would indicate an increase MNPs induced toxicity. The use of bacteria with these characteristics represent a new method to address the toxicity of different MNPs in a short time period by analyzing fluctuations of light emission [93]. This characteristic of luminous bacteria has been used in promising toxicity studies for Au nanoparticles and carbon nanotubes in just 15-minute assays [94]. The results obtained with the luminous bacteria were comparable to those obtained with other conventional approaches used to evaluate MNPs induced toxicity [95].

\section{Conclusions}

The different alternatives used to date to evaluate the potential toxicity of MNPs using both in vitro and in vivo assays has been presented. Although there are an increasing number of articles aiming to assess the toxicity of MNPs, there is still an urgent need for standardized protocols allowing for a systematic study of the toxicity induced by a particular type of MNPs. As it has been shown, not only the concentration and the exposure times are parameters that have to be considered when assessing the toxicity of MNPs, but also the stability, particle size, shape and the aggregation state are crucial factors. Moreover, when using in vitro models, it has been proved that the behavior may be radically different depending on the cell line tested. As for the in vivo models, it has also been shown the importance of choosing not only the correct animal model but also the appropriate route of administration, since the 
toxic response can greatly vary. In addition, it is important to mention that although general toxicity assays have been shown to be very useful to evaluate MNPs-induced toxicity, approaches focused on the identification of specific targets (genes and/or proteins) may help not only to better understand the interaction mechanisms of cells and organisms with MNPs, but also to design novel non-toxic MNPs.

\section{Acknowledgments}

This work was funded by grants from the Spanish Ministry of Economy and Competitiveness: CTQ201018644, CTQ2011-28328C02-01 and SAF2011-27330; and grants ANALISYC II and INDISNET 01592006 from Comunidad de Madrid. J.L.L.-G. was financially supported by the "Ramón y Cajal" program. I.L.-H. thanks the Comunidad de Madrid for a predoctoral scholarship. 


\section{References}

[1] D. Cui, H. Gao, Biotechnol. Prog. 19 (2003) 683.

[2] V. Rabolli, L.C.J. Thomassen, F. Uwambayinema, J.A. Martens, D. Lison, Toxicol. Lett. 206 (2011) 197.

[3] K. Gerloff, I. Fenoglio, E. Carella, J. Kolling, C. Albrecht, A.W. Boots, I. Förster, R.P.F. Schins, Chem. Res. Toxicol. 25 (2012) 646.

[4] R.D. Handy, R. Owen, E. Valsami-Jones, Ecotoxicology 17 (2008) 315.

[5] S.G. Mukherjee, N. O'Claonadh, A. Casey, G. Chambers, Toxicol. in Vitro 26 (2012) 238.

[6] M. Tarantola, D. Schnelder, E. Sunnick, H. Adam, S. Pierrat, C. Rosman, V. Breus, C. Sönnichsen, T. Basché, J. Wegener, A. Janshoff, ACS nano 3 (2009) 213.

[7] V. Christen, K. Fent, Chemosphere 87 (2012) 423.

[8] H.A. Ngwa, A. Kanthasamy, Y. Gu, N. Fang, V. Anantharam, A.G. Kanthasamy, Toxico. Appl. Pharm. 256 (2011) 227.

[9] M. Al-Rawi, S. Diabaté, C. Weiss, Arch. Toxicol. 85 (2011) 813.

[10] S.M. Hussain, K.L. Hess, J.M. Geahart, K.T. Geiss, J.J. Schlager, Toxicol. In Vitro 19 (2005) 975.

[11] Z.E. Allouni, P.J. Høl, M.A. Cauqui, N.R. Gjerdet, M.R. Cimpan, Toxicol. in Vitro 26 (2012) 469.

[12] M.J.D. Clift, B. Rothen-Rutishauser, D. M. Brown, R. Duffin, K. Donaldson, L. Proudfoot, K. Guy, V. Stone, Toxicol. App. Pharm. 232 (2008) 418.

[13] T. Morais, M.E. Soares, J.A. Duarte, L. Soares, S. Maia, P. Gomes, E. Pereira, S. Fraga, H. Carmo, Mde L. Bastos, Eur. J. Pharm. Biopharm. 80 (2012) 185.

[14] M. Shen, H. Cai, X. Wang, X. Cao, K. Li, S.H. Wang, R. Guo, L. Zheng, G. Zhang, X. Shi, Nanotechnology 23 (2012) 105601.

[15] A. Albanese, W.C.W. Chan, ACS nano 5 (2011) 5478.

[16] C. Brandenberger, M.J.D. Clift, D. Vanhecke, C. Mühlfeld, V. Stone, P. Gehr, B. RothenRutishauser, Particl. Fib. Toxicol. 7 (2010) 1.

[17] J.H. Park, J. Park, U. Dembereldorj, K. Cho, K. Lee, S.I. Yang, S.Y. Lee, S.W. Joo, Anal. Bioanal. Chem. 401 (2011) 1631.

[18] R. de Lima, A.B. Seabra, N. Duran, J. Appl. Toxicol. (2012) doi:10.1002/jat.2780.

[19] B.J. Marquis, S.A. Love, K.L. Braun, C.L. Haynes, Analyst 134 (2009) 425.

[20] H.E. Pace, E.K. Lesher, J.F. Ranville, Environ. Toxicol. Chem. 29 (2010) 1338.

[21] F. Watari, N. Takashi, A. Yokoyama, M. Uo, T. Akaska, Y. Sato, S. Abe, Y. Totsuka, K. Tohij, J. R. Soc. Interface 6 (2009) S371.

[22] R. Allabashi, W. Stach, A. de la Escosura-Muniz, L. Liste-Calleja, A. Merkoci, J. Nanopart. Res. 11 (2009) 2003.

[23] M.C. Wende, J.A.C. Broekaert, Fresenius' J. Anal. Chem. 370 (2001) 513.

[24] M. Resano, F. Vanhaecke, M.T.C. de Loos-Vollebregt, J. Anal. At. Spectrom. 23 (2008) 1450.

[25] A. Scheffer, c. Engelhard, M. Sperling, W. Buscher, Anal. Bioanal. Chem. 390 (2008) 249. 
[26] W. Zhang, Y. Ji2, J. Meng, X. Wu, H Xu, PLoS ONE 7 (2012) e31957.

[27] D.A. Navarro, M.A. Bisson, D.S. Aga, J. Hazardous Materials 211- 212 (2012) 427.

[28] A. Sarmiento-Gonzalez, J.R. Encinar, J.M. Marchante-Gayon, A. Sanz-Medel, Anal. Bioanal. Chem. 393 (2009) 335.

[29] J.L. Tang, L. Xiong, S. Wang, J.Y. Wang, L. Liu, J. Li, F.Q. Yuan, T.F. Xi, J. Nanosci. Nanotechnol. 9 (2009) 4924.

[30] M.M. Song, W.J. Song, H.Bi, J. Wang, W.Lin Wu, J. Sun, M. Yu, Biomaterials 31 (2010) 1509.

[31] Y. Hayashi, P. Engelmann, R. Foldbjerg, M Szabo, I. Somogyi, E. Pollák, L. Molnár, H. Autrup, D.S. Sutherland, J. Scott-Fordsmand, L.H. Heckmann, Environ. Sci. Technol. 46 (2012) 4166.

[32] F. Liu. J. Chromatogr. A 1216 (2009) 9034.

[33] K. Teide, A.B.A. Boxall, X. Wang, D. Gore, D. Tiede, M. Baxter, H. David, S.P. Tear, J. Lewis. J. Anal At. Spectrom. 25 (2010) 1149.

[34] U. Kumtabtim, J. Shiowatana, A. Siripinyanond, J. Anal. At. Spectrom 2005 (20) 1185.

[35] A. Samontha, J. Shiowatana, A. Siripinyanond, Anal. Bioanal. Chem. 399 (2011) 973.

[36] A. Oukarroum, S. Bras, F. Perreault, R. Popovic, Ecotoxicol. Environ. Safety 78 (2012) 80.

[37] S.Y. Choi, S. Jeong, S.H. Jang, J. Park, J.H. Park, K.S. Ock, S.Y. Lee, S.W. Joo, Toxicol. in Vitro 26 (2012) 229.

[38] G.D. Zhang, Z. Yang, W. Lu, R. Zhang, Q. Huang, M. Tian, L. Li, D. Liang, C. Li, Biomaterials 30 (2009) 1928.

[39] J.G. Teeguarden, P.M. Hinderliter, G. Orr, B.D. Thrall, J.G. Pounds, Toxicol. Sci. 95 (2007) 300.

[40] A.M. Alkilany, P.K. Nagaria, C.R. Hexel, T.J. Shaw, C.J. Murphy, M.D. Wyatt, Small 5 (2009) 701.

[41] J. Chan, T. Ying, Y.F. Guang, L.X. Lin, T. Kai, Z.V. Fang, Y.X. Ting, L.F. Xing, Y.Y. Ji, Biol. Trace Elem. Res. 144 (2011) 183.

[42] T. Hasezaki, K. Isoda, M. Kondoh, Y. Tsutsumi, K. Yagi, Pharmazie 66 (2011) 698.

[43] X. Lu, J.C. Qian, H.J. Zhou, Q. Gan, W. Tang, J.X. Lu, Y. Yuan, C.S. Liu, Int. J. Nanomedicine 6 (2011) 1889.

[44] H. Luo, F. Wang, Y. Bai, T. Chen, W. Zheng, Colloids Surf. B Biointerfaces 94 (2012) 304.

[45] H.K. Patra, S. Banerjee, U. Chaudhuri, P. Lahiri, A.K. Dasgupta, Nanomed. Nanotechnol. 3 (2007) 111.

[46] R.M. Zucker, E.J. Massaro, K.M. Sanders, L.L. Degn, W.K. Boyes, Cytometry Part A 77A (2010) 677.

[47] X. Han, N. Corson, P. Wade-Mercer, R. Gelein, J. Jiang, M. Sahu, P. Biswas, J.N. Finkelstein, A. Elder, G. Oberdörster, Toxicology 297 (2012) 1.

[48] C. Uboldi, G. Giudetti, F. Broggi, D. Gilliland, J. Ponti, F. Rossi, Mutat. Res. 745 (2012) 11.

[49] J.-H. Yuan, Y. Chen, H.-X. Zha, L.-J. Song, C.-Y. Li, J.-Q. Li, X.-H. Xia, Colloids Surf. B Biointerfaces 76 (2010) 145.

[50] C. Harris, J.M. Hansen, Methods Mol. Biol. 889 (2012) 325.

[51] M. Könczöl, S. Ebeling, E. Goldenberg, F. Treude, R. Gminski, R. Gieré, B. Grobéty, B. RothenRutishauser, I. Merfort, V. Mersch-Sundermann, Chem. Res. Toxicol. 24 (2011) 1460. 
[52] I. Passagne, M. Morille, M. Rousset, I. Pujalté, B. L’Azou, Toxicology 299 (2012) 112.

[53] J.J. Li, D. Hartono, C.-N. Omg, B.-H. Bay, L.-Y.L. Yung, Biomaterials 31 (2010) 5996.

[54] V. Sharma, D. Anderson, A. Dhawan, Apoptosis 17 (2012) 852.

[55] E.-J. Park, J. Yi, K.-H. Chung, D.-Y. Ryu, J. Choi, K. Park, Toxicol. Lett. 180 (2008) 222.

[56] J. Ahmad, M. Ahamed, M.J. Akhtar, S.A. Alrokayan, M.A. Siddiqui, J. Musarrat, A.A. A1Khedhairy, Toxicol. Appl. Pharm. 259 (2012) 160.

[57] M. van Gurp, N. Festjens, G. van Loo, X. Saelens, P. Vandenabeele, Biochem. Biophys. Res. Commun. 304 (2003) 487.

[58] T. Chen, Y.-S. Wong, W. Zheng, Y. Bai, L. Huang, Coll. Surf. B Bioinformatics 67 (2008) 26.

[59] K. Meyer, P. Rajanahalli, M. Ahamed, J.J. Rowe, Y. Hong, Toxicol. in Vitro, 25 (2011) 1721.

[60] S. Cotelle, J.F. Férard, Environ. Mol. Mutagen. 34 (1999) 246.

[61] R. Meena, M. Rani, R. Pal, P. Rajamani, Appl. Biochem. Biotechnol. 167 (2012) 791.

[62] C. Gong, G. Tao, L. Yang, J. Liu, H. He, Z. Zhuang, Mol. Biol. Rep. 39 (2012) 4915.

[63] G. Speit, P. Schütz, I. Bonzheim, K. Trenz, H. Hoffmann, Toxicol. Lett. 146 (2004) 151.

[64] N. Hanagata, F. Zhuang, S. Connolly, J. Li, N. Ogawa, M. Xu, ACS nano 5 (2011) 9326.

[65] M. Mahmoudi, S. Laurent, M.A. Shokrgozar, M. Hosseinkhani, ACS nano 5 (2011) 7263.

[66] P. Dua, K.N. Chaudhari, C.H. Lee, N.K. Chaudhari, S.W. Hong, J.-S. Yu, S. Kim, D.-K. Lee, Bull. Korean Chem. Soc. 32 (2011) 2051.

[67] J.L. Luque-Garcia, P. Cabezas-Sanchez, C. Camara, Trends Anal. Chem. 30 (2011) 703.

[68] J.L. Luque-Garcia, J.L. Martinez-Torrecuadrada, C. Epifano, I. Babel, M. Cañamero, I. Casal, Proteomics $10(2010) 940$.

[69] X. Yang, J. Liu, H. He, L. Zhou, C. Gong, X. Wang, L. Yang, J. Yuan, H. Huang, L. He, B. Zhang, Z. Zhuang, Part. Fibre Toxicol. 7 (2010) 1.

[70] Y.-M. Jeon, S.-K. Park, M.-Y. Lee, J. Korean Soc. Appl. Biol. Chem. 54 (2011) 852.

[71] C.A. Simpson, K.J. Salleng, D.E. Cliffel, D.L. Feldheim, Nanomedicine (2012) In press.

[72] K.L. Aillon, Y. Xie, N. El-Gendy, C.J. Berkland, M.L. Forrest, Adv. Drug Deliv. Rev. 61 (2009) 457.

[73] V. Kumar, A. Kumari, P. Guleria, S.K. Yadav, Rev. Environ. Cont. Toxicol. 215 (2012) 39.

[74] R.J. Griffitt, J. Luo, J. Gao, J.C. Bonzongo, D.S. Barber, Environ. Toxico.1. Chem. 27 (2008) 1972.

[75] Y.L. Hu, W. Qi, F. Han, J.Z. Shao, J.O. Gao, Int. J. Nanomedicine 6 (2011) 3351.

[76] J.H. Sung, J.H. Ji, J.D. Park, M.Y. Song, K.S. Song, H.R. Ryu, J.U. Yoon, K.S. Jeon, J. Jeong, B.S. Han, Y.H. Chung, H.K. Chang, J.H. Lee, D.W. Kim, B.J. Kelman, I.J. Yu, Part. Fibre Toxicol. 8 (2011) 16.

[77] J.S. Kim, T.J. Yoon, K.N. Yu, B.G. Kim, S.J. Park, H.W. Kim, K.H. Lee, S.B. Park, J.K. Lee, M.H. Cho, Toxicol. Sci. 89 (2006) 338.

[78] W.H. De Jong, W.I. Hagens, P. Krystek, M.C. Burger, A.J. Sips, R.E. Geertsma, Biomaterials 29 (2008) 1912. 
[79] H. Xie, M.M. Mason, J.P. Wise, Rev. Environ. Health 26 (2011) 251.

[80] G. Oberdorster, E. Oberdorster, J. Oberdorster, Environ. Health Perspect. 113 (2005) 823.

[81] G.M. Hodges, E.A. Carr, R.A. Hazzard, C. O'Reilly, K.E. Carr, J. Drug Target 3 (1995) 57.

[82] Z. Chen, H. Meng, G. Xing, C. Chen, Y. Zhao, G. Jia, T. Wang, H. Yuan, C. Ye, F. Zhao, Z. Chai, C. Zhu, X. Fang, B. Ma, L. Wan, Toxicol. Lett. 163 (2006) 109.

[83] S.K. Lam, Clin. Gastroenterol. 14 (2000) 41.

[84] S. Novak, D. Drobne, A. Menard, Zookeys 176 (2012) 261.

[85] A. Jemec, D. Drobne, M. Remskar,K. Sepcic, T. Tisler, Environ. Toxicol. Chem. 27 (2008) 1904.

[86] D. Drobne, A. Jemec, Z. Pipan Tkalec, Environ. Pollut. 157 (2009) 1157.

[87] S. Asghari, S.A. Johari, J.H. Lee, Y.S. Kim, Y.B. Jeon, H.J. Choi, M.C. Moon, L.J. Yu, J. Nanobiotechnology 10 (2012) 14.

[88] A.J. Kennedy, M.S. Hull, A.J. Bednar, J.D. Goss, J.C. Gunter, J.L. Bouldin, P.J. Vikesland, J.A. Steevens, Environ. Sci. Tech. 44 (2010) 9571.

[89] H.J. Allen, C.A. Impellitteri, D.A. Macke, J.L. Heckman, H.C. Poynton, J.M. Lazorchak, S. Govindaswamy, D.L. Roose, M.N. Nadagouda, Environ. Toxicol. Chem. 29 (2010) 2742.

[90] S. Zhu, E. Oberdorster, M.L. Haasch, Marine Environ. Res. 62 (2006) S5.

[91] A.A. Clancy, Y. Gregoriou, K. Yaehne, D.R. Cramb, Chem. Phys. Lett. 488 (2010) 99.

[92] M.Y. Wani, M.A. Hashim, F. Nabi, M.A. Malik, Adv. Phys. Chem. 2011 (2011) 1-15.

[93] S. Parvez, C. Venkataraman, S. Mukherji, Environ. Int. 32 (2006) 265.

[94] H. Zheng, L. Liu, Y. Lu, Y. Long, L. Wang, K.P. Ho, K.Y. Wong, Anal. Sci. 26 (2010) 125-128.

[95] J. Lappalainen, R. Juvonen, J. Nurmi, M. Karp, Chemosphere 45 (2001) 635. 


\section{Figure Captions}

Fig. 1. URI images of L929 fibroblasts at 100x magnification after $24 \mathrm{~h}$ exposure to $0.5 \mathrm{mg} / \mathrm{L}$ of $\mathrm{TiO}_{2}$ NPs. Bright spots are nanoparticles either internalized or attached to the membrane. (A) Control cells. (B) Rutile, $40 \mathrm{~nm}$. (C) Rutile and anatase mixture, < $100 \mathrm{~nm}$ (D) Anatase, $10 \mathrm{~nm}$. White arrows (C) show agglomerates that appear to be attached to the cell membrane; red arrows point at agglomerates inside the cells (Reproduced with permission of Elsevier).

Fig. 2. Cytotoxicity of nanoparticles. Huh7 (left) and FHM (right) cells were treated for $24 \mathrm{~h}$ with different concentrations of $\mathrm{SiO}_{2}-\mathrm{NPs}$ (blue dots); $\mathrm{SiO}_{2}-1 \%$ Ag-NPs (green squares), $\mathrm{SiO}_{2}-5 \%$ Ag-NPs (black triangle); and $\mathrm{SiO}_{2}-20 \% \mathrm{Ag}-\mathrm{NPs}$ (red triangle) followed by assessing cell viability by MTT assay. Shown are the results of three independent experiments. (Reproduced with permission of Elsevier).

Fig. 3. The effects of exposure to $15,30,100 \mathrm{~nm}$ or micro-sized $\mathrm{SiO}_{2}$ particles on $\mathrm{DNA}$ damage in $\mathrm{HaCat}$ (human keratinocytes) cells. Representative images and quantitative analysis of tail length or tail DNA percentage of comet in $\mathrm{HaCaT}$ cells. Values shown were the mean from 300 randomly selected comet images of each sample. (Reproduced with permission of Springer). 